Oliver Kohl-Frey*

\title{
Bibliothek als Ort, Bibliothek als Organisation, Bibliothek im Wandel
}

\author{
Räumliche, funktionelle und organisatorische Veränderungen an der Universität Konstanz im \\ Vergleich zum Gründungskonzept
}

DOI 10.1515/bfp-2016-0046

Zusammenfassung: Die Bibliothek der Universität Konstanz setzt seit Gründung der Universität vor 50 Jahren auf Einschichtigkeit, systematische Freihandaufstellung und Benutzerorientierung. In den letzten fünf Jahren wurden große Teile des Bibliotheksgebäudes schadstoffsaniert. Während dieser Sanierung wurde das der Bibliothek zugrundeliegende Konzept neu bewertet und auf die aktuelle Situation und die Bedürfnisse an der Hochschule angepasst. Der Artikel beschäftigt sich mit den räumlichen und organisatorischen Veränderungen im Verhältnis zum Gründungskonzept der Bibliothek.

Schlüsselwörter: Universitätsbibliothek; Konstanz; Funktion; Wandel; Reorganisation

\section{Library as Space, Library as Organization, Library in the Change Process. Spatial, Functional and Organizational Changes at the University of Konstanz in Relation to the Founding Concept}

Abstract: Since the founding of the University of Konstanz 50 years ago the library stands for a centralized library system, free access to systematically arranged media and user orientation. During the last five years large parts of the building have been renovated and the renovation process of the library founding concept has been re-evaluated and adapted to the current situation and needs. This article deals with the spatial and organizational changes in relation to the founding concept.

Keywords: University library; Konstanz; function; change; reorganization

\section{Einleitung}

Joachim Stoltzenburg, der erste Leitende Bibliotheksdirektor der 1966 gegründeten Universität Konstanz, schreibt rückblickend über die der Bibliothek zugrundeliegende Konzeption: „Die Gründung neuer Hochschulen und ihrer Bibliotheken eröffnete uns eine Chance, die Fragen der Literaturversorgung einer Hochschule neu zu denken." ${ }^{1}$

Diese Chance wurde im Konstanzer Gründungsprozess radikal genutzt. Unter Stoltzenburgs Führung wurden zentrale Innovationen wie die strikte Einschichtigkeit des Bibliothekssystems ohne dezentrale Bibliotheken und die systematische Freihandaufstellung aller Bestände nach der neu entwickelten Konstanzer Systematik konsequent umgesetzt. In den folgenden Jahrzehnten wurden, teils schon unter seinen Nachfolgern, weitere Schritte realisiert, die zunächst von außerhalb kritisch beäugt wurden, sich aber mittlerweile fast flächendeckend durchgesetzt haben, wie etwa die integrierte Medienbearbeitung in den achtziger Jahren oder die Einführung der ersten 24-Stunden-Bibliothek im deutschsprachigen Raum im Jahre 2001. ${ }^{2}$

In den vergangenen Jahren wurde die Bibliothek der Universität grundlegend räumlich saniert, nachdem Teile des Gebäudes nach Asbestfunden im November 2010 geschlossen werden mussten. Doch wurde dabei nicht nur Schadstoffbeseitigung betrieben, sondern auch das der Bibliothek zugrundeliegende Raum- und Funktionskonzept einer gründlichen Prüfung und Überarbeitung unterzogen. Hierbei handelte es sich nun zwar keineswegs um eine Neugründung, aber dennoch um eine einmalige Chance, das Bibliothekskonzept aus den 1970er-Jahren zu überdenken und an die heutigen und zukünftigen Erfordernisse anzupassen. 


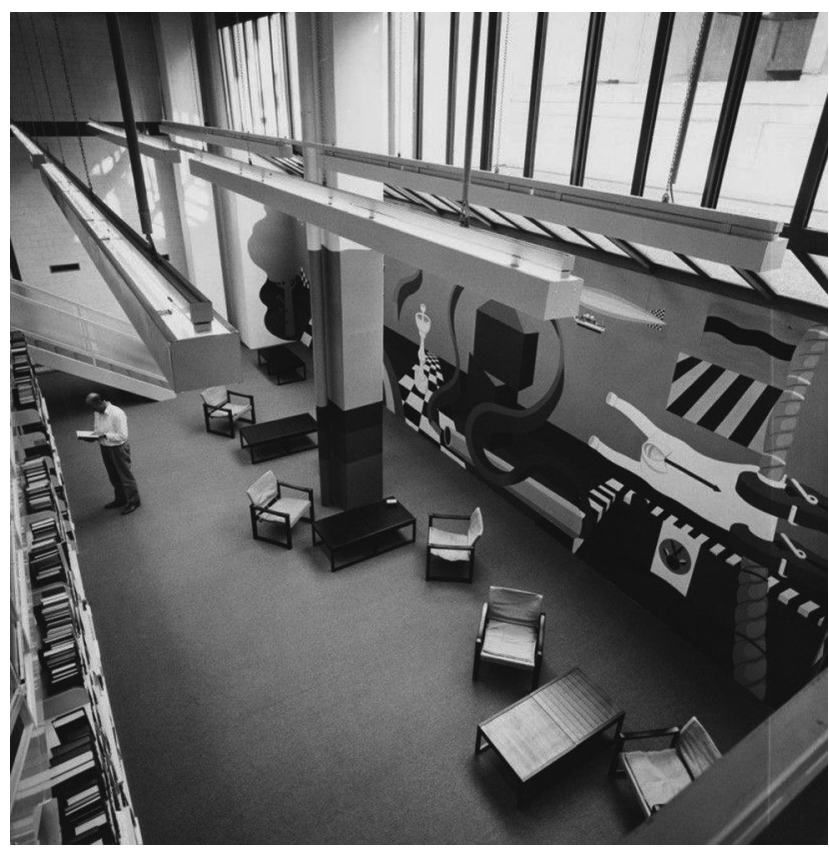

Abb. 1: Wissenschaftliches Arbeiten in den 1970er-Jahren: Arbeitsplätze direkt bei der systematischen Freihandaufstellung. Fotograf: Unbekannt, ${ }^{\odot}$ Universität Konstanz

Mit der Wiedereröffnung zweier zentraler Gebäudeteile im September 2015 wurde somit nicht nur die Schadstoffsanierung weitgehend abgeschlossen, sondern gleichzeitig die Anpassung des bibliothekarischen Gründungskonzepts an die aktuelle Hochschullandschaft vollzogen. Doch damit nicht genug: Parallel zur räumlichen Sanierung wurde die Bibliothek auch organisatorisch in einen größeren Zusammenhang, in das ab 2009 begründete Kommunikations-, Informations-, Medienzentrum (KIM) der Universität. Damit wurde die Bibliothek Teil des gesamtuniversitären Dienstleisters für die gesamte Literatur- und Informationsversorgung.

Der vorliegende Aufsatz beschreibt in erster Linie die räumliche Sanierung entlang des Stoltzenburg'schen Gründungsgedankens, bezieht aber daneben auch die funktionellen und organisatorischen Veränderungen der Bibliothek im Rahmen des KIM mit ein.

\section{Reflektion und Überarbeitung des Konzepts}

Die Stoltzenburg'schen Grundgedanken finden sich nicht in Stein gemeißelt über einem ohnehin nicht vorhandenen Eingangsportal zur Bibliothek als einer Halle des Wissens. Dies ist aber auch nicht notwendig, denn zentrale Bestandteile wie die Systematische Freihandaufstellung oder die Benutzerorientierung bilden ein gemeinsames Grundver- ständnis des Konstanzer Bibliothekskollegiums. Dieses Grundverständnis wurde zu Beginn der Sanierungszeit in mehreren bibliotheksinternen Gesprächsrunden diskutiert und weiterentwickelt. Es war darüber hinaus selbstverständlich, die Benutzer von Beginn an mit in die Konzeption der Sanierung einzubeziehen.

Dabei wurde in der Diskussion mit den universitären Entscheidungsträgern wie dem Rektorat, dem Senat mit seinem zuständigen Ausschuss für Kommunikation und Information (AKI) oder dem mit Externen besetzten Universitätsrat durchaus offen und grundsätzlich thematisiert, welche Form und Dimension von Bibliothek an der Universität Konstanz zukünftig gebraucht würde. Die von der Bibliothek vorgestellten konzeptionellen Planungen fanden dabei weitgehend die Zustimmung der beteiligten Wissenschaftler und Entscheidungsträger. Es darf sicherlich als Erfolg verbucht werden, dass die Bibliothek an einer von extremer Raumnot betroffenen Universität nach der Sanierung noch immer 25000 Quadratmeter und damit die gleiche Fläche wie vor der Sanierung misst.

Doch nicht nur die Entscheidungsträger wurden in die konzeptionellen Überlegungen mit einbezogen, sondern auch die Studierenden. In mehreren Gesprächsrunden wurde die Konzeption mit den studentischen Gremien (Fachschaften, Allgemeiner Studierendenausschuss) besprochen. Gegen Ende der konzeptionellen Phase wurde mittels einer Wandzeitung bzw. Kommentarwand allen Studierenden die Möglichkeit der Mitwirkung eröffnet, was ausgesprochen rege genutzt wurde.

Diese Vorgehensweise findet sich schon in den Gründungsjahren. Zu dieser Zeit wurde die Grundkonzeption der neuen Bibliothek v.a. im Gründungsausschuss entworfen und beschlossen und in den späteren Jahren im Bibliotheksausschuss (dem Vorläufer des AKI) verfeinert und weiterentwickelt. ${ }^{3}$

\section{Räumliche Aufteilung}

Die Konstanzer Bibliothek mit ihrer Gesamtnutzfläche von ca. 25000 Quadratmetern besteht aus fünf Gebäudeteilen, von denen die drei aus den 1970er-Jahren stammenden Kerngebäudeteile BA (Info-Zentrum), BS und BG mit drei Vierteln der Gesamtfläche (ca. 18000 Quadratmetern) von der Schließung und Asbestsanierung betroffen waren. Die beiden anderen Gebäudeteile, N und J, mit ca. 7000 Quadratmetern stammen aus den 1980er- bzw. 2000er-Jahren und dienten während der Sanierungszeit als eingeschränkte

3 Stoltzenburg (1970, 74 und 76). 


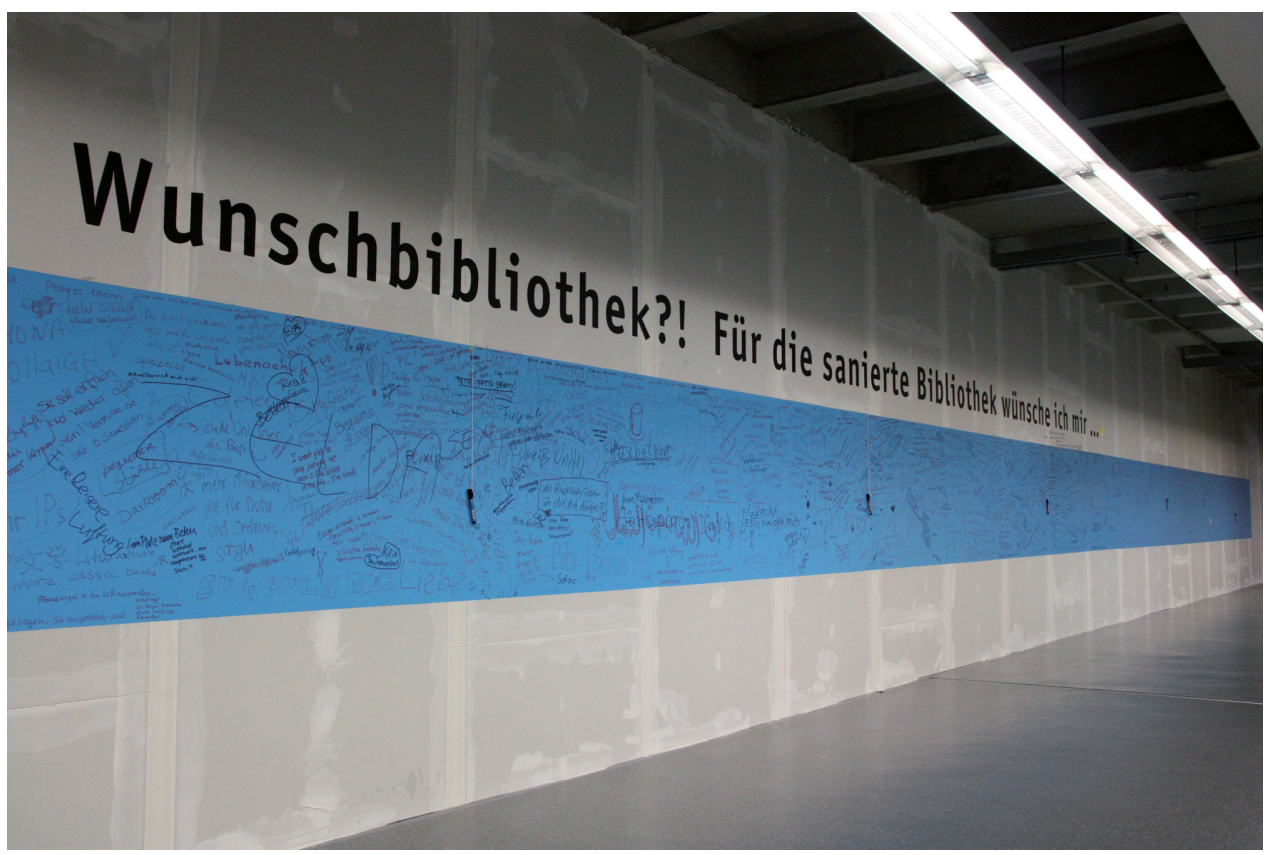

Abb. 2: Wunschbibliothek? Kommentare von Studierenden zur Sanierungsplanung an der dafür genutzten Bauwand. Fotograf: M. Müller, ${ }^{\odot}$ Universität Konstanz

Freihandbibliothek mit knapp 700 Arbeitsplätzen und 500000 Bänden. ${ }^{4}$ Daneben wurden für die Dauer der Sanierung etwa 1,5 Millionen Bände in ein separates Magazin außerhalb des Campus ausgelagert.

Die sanierten Kerngebäudeteile bilden nicht nur den Ursprung der Bibliotheksbereiche, sondern stellen auch räumlich den Mittelpunkt der Bibliothek dar. Weiter gefasst repräsentieren diese Gebäudeteile den Mittelpunkt der gesamten Reformuniversität und damit die „bibliothekarisch-organisatorische Antwort auf eine neue Universitätsstruktur". ${ }^{5}$ Das Informationszentrum mit seinen ca. 3500 Quadratmetern ist innerhalb des gesamten Bibliothekskomplexes der flexibelste Teil und der „Kern des Bibliothekssystems". ${ }^{6}$ Daran schließen sich die Buchbereiche BG, BS (und J) an, die aufgrund einer starren, selbsttragenden Stahlregalanlage kaum veränderbar sind.

Aus diesem Grund werden künftig fast ausschließlich diese Bereiche als Ort für den Bestand genutzt. Die flexiblen Flächen im Informationszentrum und in den Randbereichen hingegen sind weitgehend für die Nutzung als Lernund Arbeitsort sowie als sozialer Ort reserviert (s.u.).

Die Bibliothek im Zentrum der Universität war von den umliegenden, direkt angebauten Fachbereichsgebäuden aufgrund von umlaufenden Glaswänden schon immer sehr

$4 \mathrm{Zu}$ einer ausführlicheren Darstellung der Sanierung vgl. Kohl-Frey (2016) sowie Frank (2016).

5 Stoltzenburg $(1970,75)$.

6 Stoltzenburg $(1970,79)$. gut sichtbar. Hinsichtlich des neuen Brandschutzkonzepts und des vorgegebenen Kostenrahmens konnten diese Sichtbeziehungen zwischen Bibliothek und Fachbereichen nicht überall erhalten werden, sondern gut die Hälfte der Umgrenzungswände musste durch geschlossene Wandelemente ersetzt werden. Immerhin ein Teil aber erlaubt an den prominenten Treppenhäusern oder Gebäudeecken weiterhin den Durchblick. Die über 20 Nebeneingänge in die Bibliothek blieben erhalten, um die enge Verbindung und die kurzen Wege unter einem Dach zwischen Wissenschaft und Bibliothek weiter zu ermöglichen („Pantoffel-Entfernung“).

\section{Bestand}

Nach wie vor ist eine Bibliothek neben all ihren anderen Funktionen auch der Ort für den gedruckten Literaturbestand einer Universität, der allerdings regelmäßig gepflegt sein will, wozu auch die Aussonderung gehört. An der Universität Konstanz wird bereits seit vielen Jahren Bestandspflege durch die Fachreferenten betrieben, die in den letzten Jahren nochmals intensiviert wurde, um die oben benannte Nutzung von Flächen für studentisches Arbeiten und für soziale Zwecke zu ermöglichen. Durch die besonders intensive Bestandspflege während der Sanierungszeit

7 Stoltzenburg $(1970,77)$. 


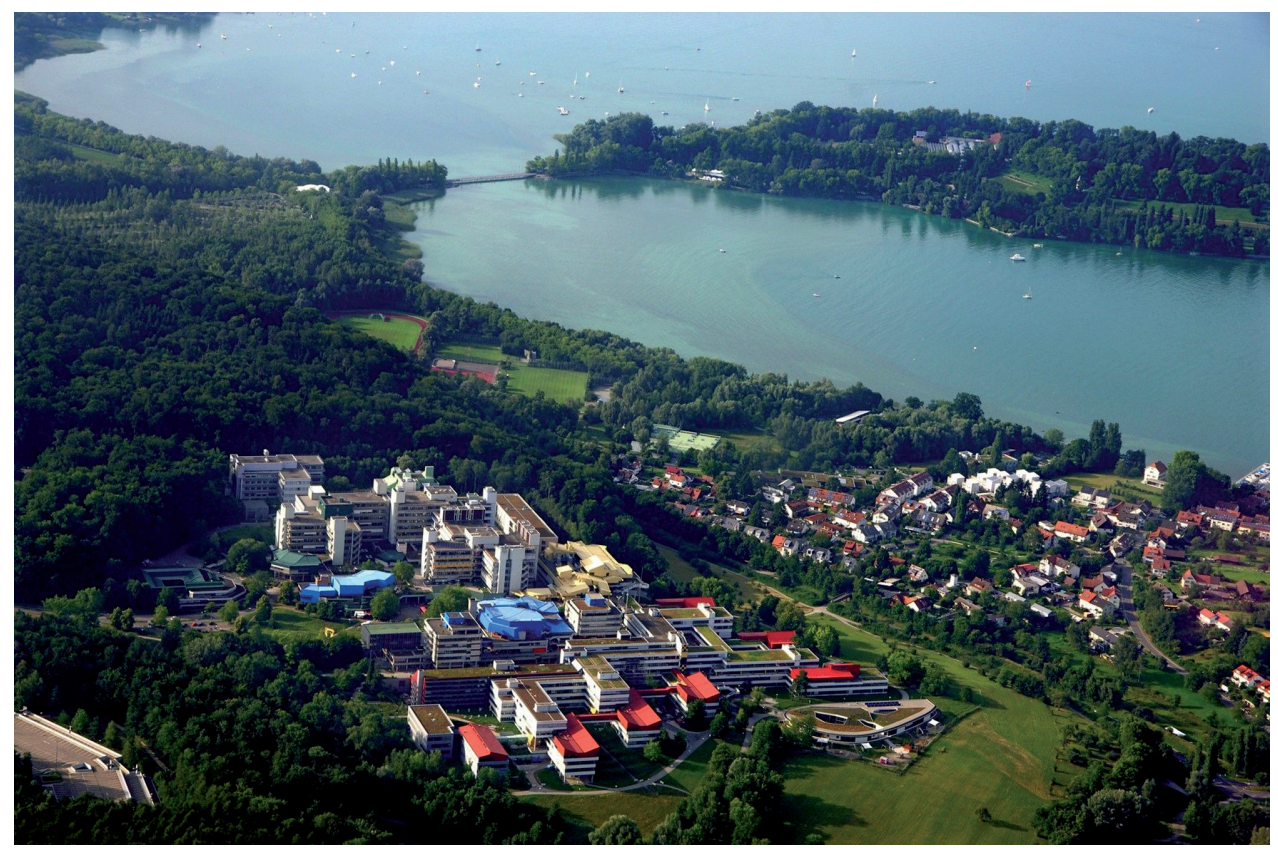

Abb. 3: Luftaufnahme der Universität Konstanz. Der zentrale Bibliotheksbereich liegt in der Form eines liegenden L östlich (unterhalb) und nördlich (rechts) des zentralen Hörsaalgebäudes (blaues Dach). Fotograf: M. Kieninger, ${ }^{\odot}$ Universität Konstanz

konnten im Zeitraum von 2010 bis 2015 insgesamt über 210000 Bände ausgesondert werden. Bei einem zeitgleichen Zugang von etwas mehr als 160000 Bänden ist die Konstanzer Bibliothek also eine schrumpfende Bibliothek, was den gedruckten Bestand angeht. Bereits Joachim Stoltzenburg legte in seinen konzeptionellen Ausführungen die Basis für diese Vorgehensweise, wenn er von veralteter „auszulesender Literatur" ${ }^{\text {" }}$ schreibt.

Im gleichen Zeitraum ist die digitale Kollektion auf knapp 150000 Titel gewachsen. ${ }^{9}$ Zwar sind diese E-Books, E-Journals und 'zig Millionen von elektronischen Aufsätzen (als in der Statistik nicht mitgeführte unselbstständige Werke) in einem Resource Discovery Service (in Konstanz auf der Basis von Summon als KonSearch angeboten) bequem findbar. Dennoch muss es eine strikte Freihandbibliothek wie Konstanz schmerzen, wenn der gesamte digitale Bestand am Ort Bibliothek überhaupt nicht sichtbar gemacht werden kann. Denn natürlich muss sich der zugrundeliegende Freihandgedanke auf den gesamten Bestand beziehen und kann sich nicht mit einer geschlossenen Magazinierung des Digitalen abfinden. Um dem zu begegnen, wurde während der Jahre 2014 und 2015 das sogenannte Hybrid Bookshelf entwickelt, eine virtuelle Suchoberfläche, mit der man in einer klassischen Regalansicht durch den

8 Stoltzenburg (1970, 89).

9 Alle Angaben: eigene Statistik. Die Zahlen sind auch in der Deutschen Bibliotheksstatistik nachzuvollziehen. digitalen (und den gedruckten) Bestand an Büchern browsen kann. ${ }^{10}$ Damit ist eine Erweiterung des Freihandzugangs auf die digitale Kollektion möglich.

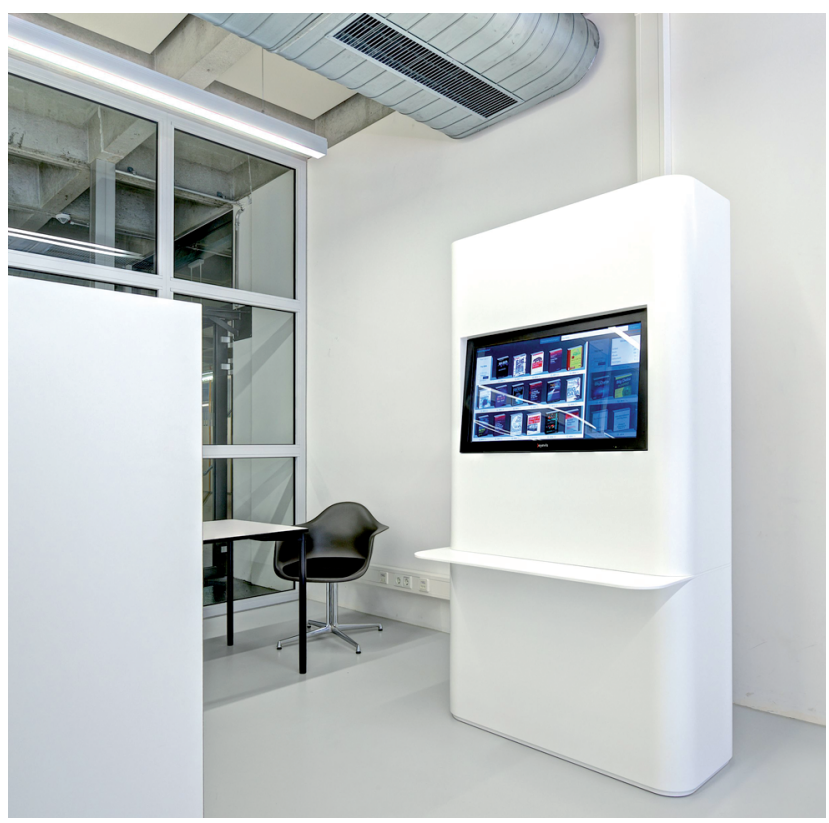

Abb. 4: Das Hybrid Bookshelf ermöglicht es, elektronischen Content am Ort Bibliothek sichtbar zu machen. Fotograf: Inka Reiter, ${ }^{\odot}$ Universität Konstanz

10 Vgl. ausführlich Kohl-Frey (2014). Die zugrundeliegende Software ist Open Source verfügbar unter www.hybridbookshelf.de. 


\section{Services}

Von Joachim Stoltzenburg ist mündlich überliefert, dass er in bibliotheksinternen Diskussionen immer wieder die Frage stellte: „Und was haben die Benutzer davon?“. Diese Benutzerorientierung lässt sich auch heute noch besonders deutlich anhand der bereitgestellten Services für die Benutzer zeigen.

Unter Klaus Franken als Leitendem Bibliotheksdirektor wurde 2001 die erste 24-Stunden-Bibliothek im deutschsprachigen Raum realisiert. ${ }^{11}$ Diese großzügigen Öffnungszeiten werden seitdem sieben Tage in der Woche kontinuierlich durchgehalten, mit Ausnahme des Zeitraums zwischen Weihnachten und Heilige Drei Könige und in den Monaten August und September. ${ }^{12}$ Diese Großzügigkeit wird von den wissenschaftlichen wie auch den studentischen Nutzern sehr geschätzt, denn damit steht die prinzipielle Möglichkeit der Bibliotheksbenutzung zu jeder Tages- und Nachtzeit zur Verfügung, wovon auch rege Gebrauch gemacht wird. Ab $21 \mathrm{Uhr}$ und am Sonntag ist dabei nur ein Wachdienst vor Ort, sodass dieser Service mit sehr geringem Personaleinsatz angeboten werden kann. Dies ist der vorausschauenden Ursprungsplanung mit nur einem zentralen Ausgang zu verdanken.

Zwar sind die digitalen Bestände für universitäre Nutzer per VPN oder Shibboleth immer auch rund um die Uhr von außerhalb des Campus zugänglich. Der Zugang zur Infrastruktur vor Ort - gedruckte Medien, Arbeitsplätze etc. wird aber erst durch die 24/7-Öffnung ermöglicht. Zusätzlich war zwar auch die Selbstausleihe über Stationen mit Barcode-Erkennung schon seit einigen Jahren möglich, aber etwas umständlich, weswegen sie von den Nutzern fast ausschließlich nachts und sonntags genutzt wurde, wenn die Ausleihtheke nicht besetzt war. Mit der Wiedereröffnung wurde nun komplett auf die sehr viel bequemere Selbstausleihe und -rückgabe mit Hilfe der RFID-Technologie umgestellt und dies auch tagsüber, wenn bibliothekarisches Fachpersonal vor Ort ist. Diese Selbstbedienung wird von den Benutzern entsprechend völlig selbstverständlich rund um die Uhr genutzt. Auch das elektronische Bezahlen von Gebühren aus dem Benutzerkonto heraus mittels Kreditkar-

11 Auch in den Anfangsüberlegungen waren die langen Öffnungszeiten schon angelegt, „wenn ein Bibliothekssystem bis tief in die Nacht hinein für seine Benutzer geöffnet sein muss“ Stoltzenburg $(1970,77)$, was für den deutschen Bibliotheksalltag Ende der 1960erJahre mehr als ungewöhnlich war.

12 Ende Dezember und Anfang Januar ist die gesamte Universität geschlossen, wovon auch die Bibliothek nicht ausgenommen werden kann. In den benutzungsschwächeren Sommermonaten ist die Bibliothek aktuell unter der Woche bis 2 Uhr nachts bzw. am Wochenende bis 23 Uhr geöffnet. te oder EC-Lastschrift ist schon einige Jahre im Einsatz. Die Schnittstelle zum Katalog ermöglicht dabei auch das Löschen einer Sperre wegen eventueller offener Bibliotheksgebühren, sodass mit Hilfe dieser gesamten Selbstbedienungsinfrastruktur unter nahezu allen Bedingungen ein selbstständiges Arbeiten des Benutzers möglich ist. Damit wird der Service der 24/7-Bibliothek nochmals deutlich erweitert und verbessert.

Der Kern des Servicekonzepts ist aber sicherlich die bibliothekarische Beratungskompetenz. In der Bibliothek der Universität Konstanz setzt sie sich aus mehreren Komponenten zusammen: An der Theke des Ausleihservice werden u. a. einfache Fragen etwa zu Benutzungsbedingungen, zur räumlichen Orientierung oder zu Recherchen im Katalog beantwortet. Die bibliothekarische Information steht für den größten Teil aller fachlichen Fragen zur Verfügung, womit weiterhin gilt, was schon Stoltzenburg schrieb, dass „Diplom-Bibliothekare [...] dort jedem Ratsuchenden behilflich sein “13 werden. Bei speziellen fachlichen Fragen werden die Fachreferenten konsultiert, die in Konstanz als einschichtiges System schon immer eine wichtige Scharnierfunktion zwischen Bibliothek und Fachwissenschaftlern haben.

Mit der Wiedereröffnung im September 2015 allerdings wurde die bibliothekarische Theke im Info-Zentrum der Bibliothek zu einer KIM-Beratungstheke ausgebaut: Neben der bibliothekarischen Information steht dort jetzt wochentags zu den gleichen Zeiten (9-19 Uhr) der universitäre ITSupport für alle Frage rund um Netze, Textverarbeitung oder Probleme mit Endgeräten aller Art bereit. Und eine zweite Erweiterung des Beratungsangebots findet sich jetzt im Info-Zentrum als dem Herzen der Bibliothek: Die universitäre Schreibberatung hilft bei allen Fragen zu Zeitmanagement, Textverarbeitung oder konzeptionellem Vorgehen im Schreibprozess. Damit sind in der Bibliothek nahezu alle einschlägigen Beratungsstellen für Studierende vom Recherchekonzept über die technische Ausstattung bis hin zum wissenschaftlichen Schreiben und der Literaturverwaltung konzentriert.

\section{Raum zum Lernen und sozialer Ort}

Bezogen auf die für die in den 1960er- und 1970er-Jahren völlig ausreichende Ausstattung der Buchbereiche mit einer „gewissen[n] Anzahl von Anleseplätzen“14 haben sich die

13 Stoltzenburg $(1970,80)$.

14 Stoltzenburg (1970, 78). Arbeiten in einer Bibliothek ließ sich zu diesem Zeitpunkt in der Mehrzahl der Fälle noch auf einen Tisch, einen Stuhl und ordentliche Beleuchtung reduzieren. 
Anforderungen an Arbeitsplätze für Nutzer spätestens seit dem Beginn der Bologna-Reform deutlich verändert. Auch die Bibliothek der Universität Konstanz hat darauf frühzeitig reagiert und etwa in dem 2003 eröffneten J-Gebäude bereits 54 Räume integriert, die flexibel sowohl für Einzelals auch für Gruppenarbeit genutzt werden können. Mit der Wiedereröffnung wurde allergrößter Wert auf den Erhalt bestehender und die Schaffung zusätzlicher flexibler Flächen geschaffen, was teilweise durch die weiter oben beschriebene Bestandspflege erreicht wurde.

Auf diesen Flächen wurde nun eine Vielzahl sehr verschiedener Arbeitsplatz-Szenarien realisiert. Ruhige Einzelarbeitsplätze werden von vielen Nutzern nach wie vor sehr geschätzt - in dieser Beziehung hat sich gegenüber den späten 1960er-Jahren nichts verändert (außer dass heute auch noch Strom und Internet benötigt werden).

Darüber hinaus aber wurden weitere Gruppenarbeitsbereiche und -räume geschaffen, in der Regel mit technisch hochwertiger Ausstattung wie großen Displays mit TouchFunktion zum Anschluss mitgebrachter Geräte, aber selbstverständlich auch Basis-Arbeitshilfen wie mobilen Whiteboards. Eine Vielzahl eher unkonventioneller Möbel ermöglicht den Benutzern im Info-Zentrum, dass jeder den für ihn passenden Platz findet. Medien-Arbeitsplätze wie BuchScanner, Micro-Film-Scanner, Videoschnitt- oder AudioPlätze gehören zur Ausstattung der seit den 1980er-Jahren aufgebauten Mediothek. Zwei Filmräume für das gemeinsame Schauen und Analysieren von Filmen, etwa in medienwissenschaftlichen Seminaren, bereichern dort das Angebot.

Im Gegensatz zu der bisherigen Wahrnehmung einer Bibliothek als einem Ort des ruhigen Arbeitens setzt sich mehr und mehr auch die Rolle als sozialer und kommunikativer Ort durch. Bibliotheken werden auch für Studierende zum „dritten Ort“, neben dem Zuhause und dem Hörsaal. Wenn allerdings Bibliotheken nicht mehr nur stille, sondern auch lebendige Räume sind, ändern sich auch die Anforderungen an Akustik, Beleuchtung, Möblierung und angebotenen Services. Neben den bereits beschriebenen Diensten ist an dieser Stelle noch das neue Café innerhalb der Bibliothek zu nennen, das neben kontemplativer Kaffeehaus-Atmosphäre auch einen Ort für das Essen und Trinken bietet.

Grundsätzlich wurden alle Arbeitsbereiche flächendeckend und großzügig mit Steckdosen, LAN-Buchsen sowie Wi-Fi ausgestattet. Für die kabelgebundene LAN-Versorgung, die v.a. für Anwendungen mit höherem Bedarf an Bandbreite wie etwa Streaming benötigt werden, werden Patchkabel über die Beratungstheke ausgeliehen. Dort können Benutzer auch Notebooks und Tablets ausleihen.
„Die neue Informationstechnik, vor allem der Einsatz des Computers, zusammen mit neuen Unterrichtsmedien und der Veränderung der Hochschuldidaktik, weisen auf nicht übersehbare Entwicklungen im Lehr- und Informationswesen hin, die die funktionsgerechte Bibliotheksplanung nachhaltig beeinflussen werden." ${ }^{15}$

In diesem Satz eines Bibliothekars aus dem Jahr 1970 steckt eine enorme konzeptionelle Weitsicht. Im Rahmen der Sanierung der letzten Jahre wurde nun ganz praktisch versucht, auch die Anforderungen der kommenden Jahre abzudecken. So wurde etwa die Netz- und Medientechnikinfrastruktur entsprechend modernisiert (Glasfaseranbindung der einzelnen Gebäude, Digitalisierung der Medientechnik aller entsprechenden Räume etc.). Mit zwei sogenannten Labs wurden Räume geschaffen, in denen zukünftige Entwicklungen und Services im Beta-Status ausprobiert und getestet werden können, die vielversprechend sein könnten, aber auch wieder verworfen werden dürfen. Während mit dem Teaching Lab eher ein Angebot für digital unterstützte Lehre entsteht, sind im Media Lab derzeit eher spielerische Anwendungen zu finden wie die Untersuchung sozialer Aspekte des Gamings an einer Playstation im Rahmen eines medienwissenschaftlichen Seminars oder die Entwicklung einer Kompositions-App durch die Südwestdeutsche Philharmonie. Dieses „trial and error“ wurde ebenfalls bereits in den Anfangsjahren mitgedacht: „Die Bibliothek kann ihren Weg zu einem automatisierten und technisierten Dienstleistungs- und Informationssystem, dessen Umrisse noch nicht hinreichend genau bestimmbar sind, zu einem erheblichen Teil nur mit Hilfe des Experiments finden." ${ }^{\prime 16}$

\section{Die Reorganisation der Bibliothek hin zum KIM}

Wenn Stoltzenburg im Jahre 1970 konstatiert, es liege nahe, die „in der Organisationswissenschaft und Wirtschaft [...] entwickelten und bewährten Grundsätze der zwischenbetrieblichen Kooperation und Organisation auf die Universität anzuwenden “ ${ }^{17}$ so bezieht er das auf die neuartige Konstruktion der bibliothekarischen Einschichtigkeit an der Universität Konstanz. Diese ist nach wie vor Realität, muss aber aus heutiger Sicht organisatorisch weiter gedacht werden. Deswegen wurde unter der aktuellen, der dritten Leiten-

15 Stoltzenburg $(1970,91)$. 16 Stoltzenburg $(1970,91)$. 17 Stoltzenburg $(1970,75)$. 


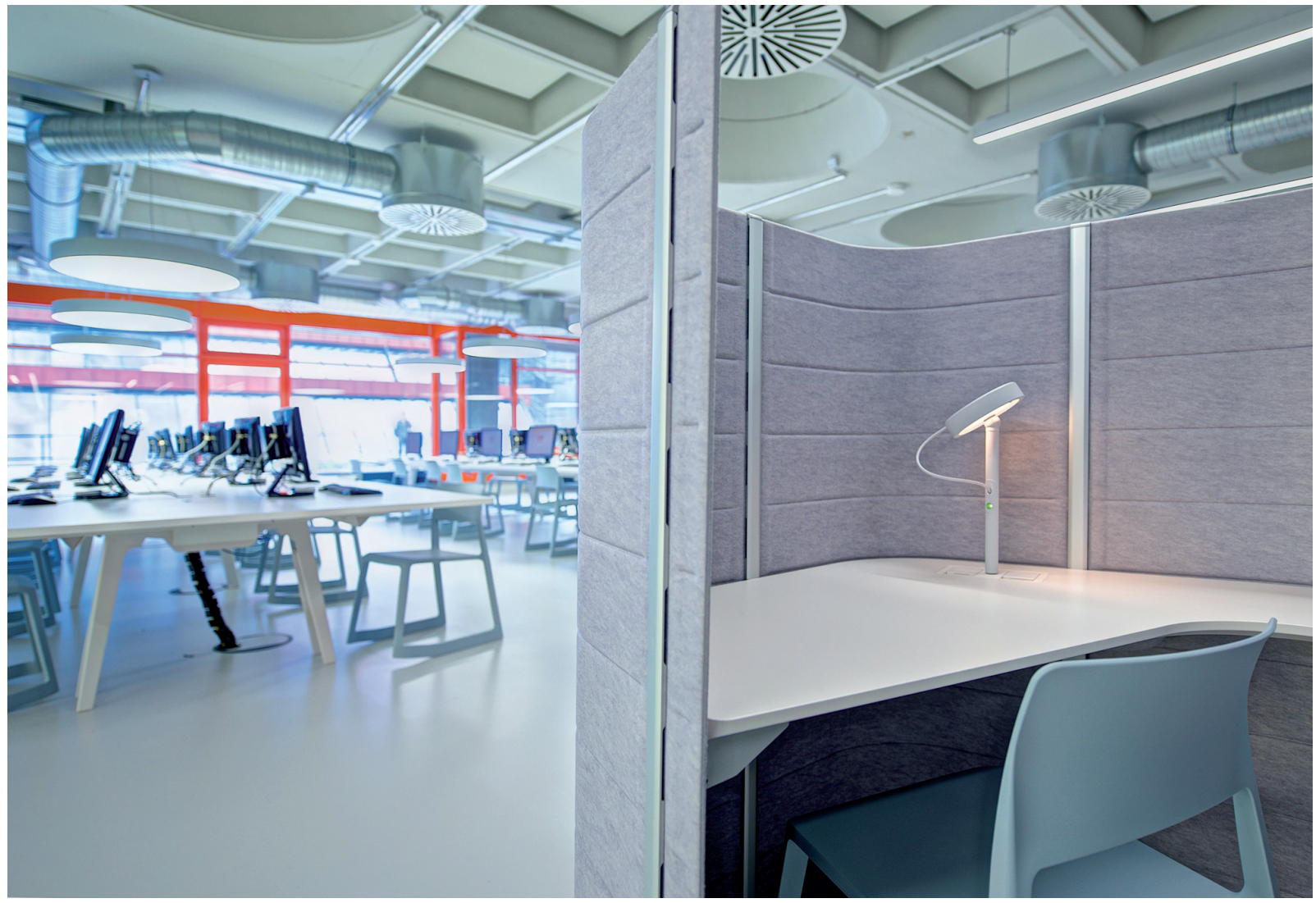

Abb. 5: Ruhige Einzelarbeitsplätze in flexiblen Lerninseln. Foto: Inka Reiter, ${ }^{\odot}$ Universität Konstanz

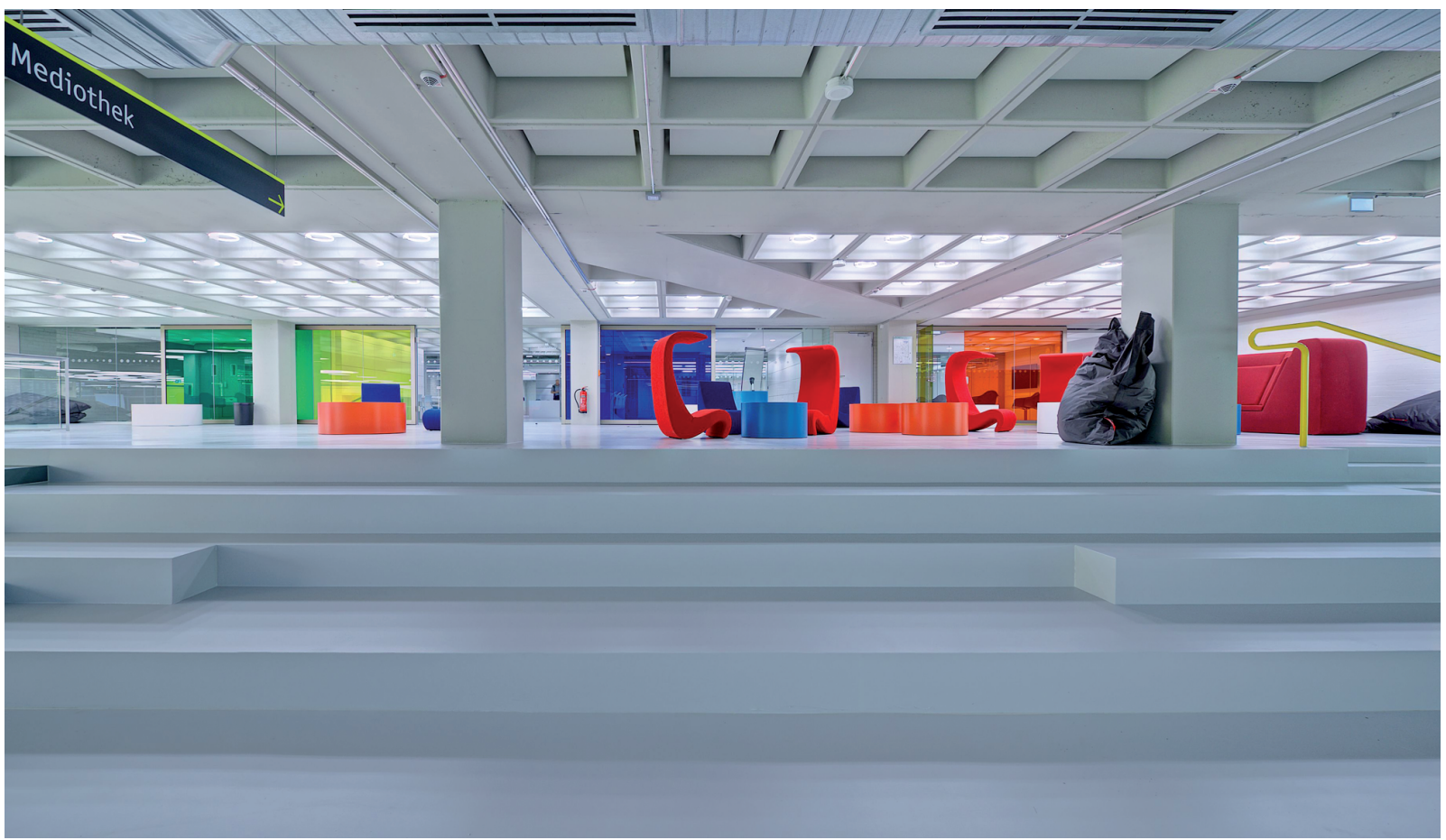

Abb. 6: Verschiedenste Sitzmöbel auf dem Medio-Deck im Info-Zentrum. Die fröhliche Farben- und Formenwelt der 1960er- und 1970er-Jahre wurde deutlich sichtbar aufgegriffen. Foto: Inka Reiter, ${ }^{\odot}$ Universität Konstanz 
den Bibliotheksdirektorin, Petra Hätscher, die verstärkte Kooperation und schließlich 2014 die organisatorische Verschmelzung der Bibliothek mit dem Rechenzentrum und der EDV-Abteilung der Universitätsverwaltung zu einem einzigen Kommunikations-, Informations-, Medienzentrum (KIM) betrieben, das heute für die gesamte Informationsund IT-Versorgung der Universität zuständig ist. Damit wird das Credo der möglichst optimalen und gleichzeitig effizienten Bereitstellung von Services für Wissenschaftler und Studierende auch auf die IT-Services übertragen. Auch hier handelt es sich um ein Experiment im Stoltzenburg'schen Sinne, ohne welches Entwicklung nicht möglich ist.

\section{Fazit}

Was ist von der ursprünglichen Konstanzer Bibliothekskonzeption, wie sie Stoltzenburg in seinem wegweisenden Aufsatz von 1970 entwickelt und reflektiert hatte, im Jahre 2016 - in dem die Universität Konstanz ihren fünfzigsten Geburtstag feiert - noch zeitgemäß? Und welche Aspekte mussten verändert, welche verworfen werden?

Nach wie vor hat die räumliche Konzeption der Konstanzer Bibliothek mit ihrem zentralen Bibliotheksgebäude und der zugehörigen systematischen Freihandaufstellung ihre Berechtigung. Auch wenn man sich heute etwa die starre und statisch selbsttragende Stahlregalanlage an einigen Stellen flexibler wünschen würde, die hauseigene Systematik sowohl Fluch als auch Segen ist oder die Freihandaufstellung um die Darstellung der digitalen Kollektion ergänzt werden musste, so steht das grundsätzliche räumliche und funktionelle Konzept außer Frage. Auch die heute geforderten Arbeitsplatz- und Serviceanforderungen lassen sich aus dem Ursprungskonzept weiterentwickeln. Das Prinzip, den größten Teil der Arbeitsplätze dezentral bei den Beständen anzuordnen, wurde auch nach der Sanierung bewusst beibehalten.

Die aus der Unzufriedenheit mit der bis dato herkömmlichen Bibliotheksorganisation resultierenden organisatorischen Neuerungen der Gründungszeit haben sich bis heute zweifellos bewährt. Die Vorteile der Einschichtigkeit des Bibliothekssystems mit seiner zentralisierten Budgetund Personalverantwortung wurde in den letzten Jahrzehnten von zahllosen ehemals zweischichtigen Systemen unter dem Stichwort der zumindest funktionalen Einschichtigkeit zu kopieren versucht. In Konstanz wurde es im Prozess des organisationalen Wandels hin $\mathrm{zu}$ einem gemeinsamen Informations- und IT-Dienstleister (dem KIM) weiterentwickelt. Der von Beginn an praktizierte Einsatz der IT als Hilfsmittel effizienten Arbeitens kann hier als früher Baustein gelten, ebenso die erstaunlich frühe Suche nach einem „Informationsingenieur“ als „neuen Typus des Bibliothekars". ${ }^{18}$

Die Weitsicht des bibliothekarischen Gründungsteams um Joachim Stoltzenburg hat zu sehr vielen richtigen Weichenstellungen beigetragen, die die zeitgemäße bibliothekarische Weiterentwicklung an der Universität Konstanz bis heute ermöglichen.

\section{Literaturverzeichnis}

Frank, Michael B. (2016): Sanierung und Neukonzeption bestehender Bausubstanz. In: Praxishandbuch Bibliotheksbau, hg. v. Petra Hauke und Klaus Werner, 136-57. Berlin: De Gruyter.

Kohl-Frey, Oliver (2014): Die Öffnung der digitalen Magazinbibliothek. Entwicklung an der Universität Konstanz zwischen Summon und Hybrid Bookshelf. In: Bibliotheksdienst, 48 (10).

Kohl-Frey, Oliver (2016): Modernisierung und Sanierung von Bibliotheksbauten. In: Praxishandbuch Bibliotheksbau, hg. v. Petra Hauke und Klaus Werner, 124-35. Berlin: De Gruyter.

Siems, Renke (2014): Innere und äußere Kreise. In: Bibliotheksdienst, $48(8 / 9), 612-32$.

Stoltzenburg, Joachim (1970): Die Bibliothek als Literaturversorgungssystem der Universität Konstanz. In: Konstanzer Blätter für Hochschulfragen, 8 (4), 74-93.

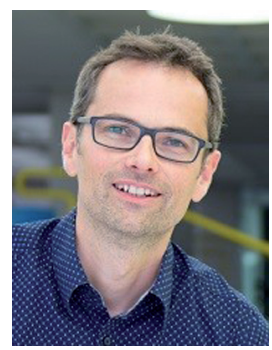

\section{Oliver Kohl-Frey}

Bibliotheksdirektor

Universität Konstanz

Kommunikations-, Informations-, Medien-

zentrum (KIM)

Universitätsstr. 10

D-78457 Konstanz

oliver.kohl@uni-konstanz.de

18 Stoltzenburg $(1970,91)$. 